\title{
Off-diagonal long-range order, cycle probabilities, and condensate fraction in the ideal Bose gas
}

\author{
Maguelonne Chevallier* and Werner Krauth ${ }^{\dagger}$ \\ CNRS-Laboratoire de Physique Statistique \\ Ecole Normale Supérieure; 24 rue Lhomond, \\ 75231 Paris Cedex 05, France
}

(Dated: October 10, 2018)

\begin{abstract}
We discuss the relationship between the cycle probabilities in the path-integral representation of the ideal Bose gas, off-diagonal long-range order, and Bose-Einstein condensation. Starting from the Landsberg recursion relation for the canonic partition function, we use elementary considerations to show that in a box of size $L^{3}$ the sum of the cycle probabilities of length $k \gg L^{2}$ equals the off-diagonal long-range order parameter in the thermodynamic limit. For arbitrary systems of ideal bosons, the integer derivative of the cycle probabilities is related to the probability of condensing $k$ bosons. We use this relation to derive the precise form of the $\pi_{k}$ in the thermodynamic limit. We also determine the function $\pi_{k}$ for arbitrary systems. Furthermore we use the cycle probabilities to compute the probability distribution of the maximum-length cycles both at $T=0$, where the ideal Bose gas reduces to the study of random permutations, and at finite temperature. We close with comments on the cycle probabilities in interacting Bose gases.
\end{abstract}

\section{INTRODUCTION}

The canonic partition function of $N$ ideal bosons in a system with energy levels $\epsilon_{0}=0<\epsilon_{1}<\ldots$ at inverse temperature $\beta=1 /\left(k_{\mathrm{B}} T\right)$ is given by

$$
Z_{N}=\left[\prod_{\sigma} \sum_{n_{\sigma}=0}^{N}\right] \exp \left(-\beta \sum_{\sigma} n_{\sigma} \epsilon_{\sigma}\right) \delta_{\sum_{\sigma} n_{\sigma}, N}
$$

For each state $\sigma$, the allowed occupation numbers $n_{\sigma}$ go from 0 to $N$, and the Kronecker $\delta$-function enforces the total number of particles to be $N$.

The Feynman path-integral [1] represents $Z_{N}$ as a trace over the diagonal density matrix $\rho^{\text {bos }}$,

$Z_{N}=\int \mathrm{d} x_{1} \ldots \mathrm{d} x_{N} \rho^{\mathrm{bos}}\left(\left\{x_{1}, \ldots, x_{N}\right\},\left\{x_{1}, \ldots, x_{N}\right\}, \beta\right)$,

which is given in terms of the $N$ ! permutations $P$ of the distinguishable-particle density matrix $\rho$

$$
\begin{aligned}
\rho^{\mathrm{bos}} & \left(\left\{x_{1}, \ldots, x_{N}\right\},\left\{x_{1}^{\prime}, \ldots, x_{N}^{\prime}\right\}, \beta\right) \\
= & \frac{1}{N !} \sum_{P} \rho\left(\left\{x_{1}, \ldots, x_{N}\right\},\left\{x_{P_{1}}^{\prime}, \ldots, x_{P_{N}}^{\prime}\right\}, \beta\right) .
\end{aligned}
$$

Thus the partition function is a sum of $N$ ! permutationdependent terms, $Z_{N}=\sum_{P} Z_{P}$, and it is possible to define the probability of a permutation $P$ as

$$
\pi_{P}=\frac{Z_{P}}{Z_{N}}
$$

\footnotetext{
* Electronic address: maguelonne.chevallier@ens.fr

${ }^{\dagger}$ Electronic address: werner.krauth@ens.fr
}

Permutations can be broken up into cycles, and one may also define cycle probabilities:

$$
\pi_{k}=\frac{1}{Z_{N}} \sum_{P \in\{N, k\}} Z_{P}
$$

where $\{N, k\}$ denotes the set of all permutations where the particle $N$ belongs to a cycle of length $k$. The choice of the particle $N$ in eqn (4) is arbitrary.

In this paper, we are concerned with the characterization of the cycle probabilities $\pi_{k}$ in the ideal Bose gas and with their relation to quantities characterizing Bose-Einstein condensation, namely the off-diagonal long range order (in Section III, see also [2, 3]) and the condensate fraction (in Section IV). In fact, for arbitrary finite systems of ideal bosons, the discrete derivative of the function $\pi_{k}$ yields the probabilities for condensing $k$ bosons [4]. This relation between the cycle statistics and the condensate fraction has not been scrutinized before in detail. It allows to determine the $\pi_{k}$ from the known fluctuation properties of the ideal Bose gas. It is also possible, and very instructive, to compute the cycle probabilities $\pi_{k}$ directly via the infinite-density limit of a finite Bose gas (see Section V). Furthermore, we will exploit some of the subtleties of the concept of cycle probabilities to compute the probability distribution of the maximum-length cycle at zero temperature, where the problem reduces to a study of random permutations [5]. Our preceding analysis of the $\pi_{k}$ allows us to understand why this distribution is essentially unchanged at finite temperature, below the condensation temperature (Section VI). Finally, we briefly review some known relations between Bose condensation and the presence of infinite cycles for interacting Bose gases (Section VII). 


\section{RECURSION RELATIONS FOR THE PARTITION FUNCTION AND THE DENSITY MATRIX}

The Landsberg recursion relation [6] gives $Z_{N}$ as a sum of only $N$ terms

$$
Z_{N}=\frac{1}{N} \sum_{k=1}^{N} Z_{N-k} z_{k}
$$

where the $z_{k}=Z_{1}(k \beta)=\sum_{\sigma} \exp \left(-k \beta \epsilon_{\sigma}\right)$ are the singleparticle partition functions at inverse temperature $k \beta$. In fact, the terms appearing in eqn (5) are the cycle probabilities:

$$
\pi_{k}=Z_{N-k} z_{k} /\left(N Z_{N}\right)
$$

This is because $k$ particles on a permutation cycle of length $k$ at inverse temperature $\beta$ have the same statistical weight $z_{k}$ as a single particle at inverse temperature $k \beta$ and, furthermore, in the ideal Bose gas, the different cycles of a permutation are statistically independent. Thus, in eqn (6) the cycle contributes $z_{k}$ and the remaining $N-k$ particles contribute $Z_{N-k}$ (see [7]).

The off-diagonal single-particle density matrix

$$
\begin{aligned}
& \rho_{N}\left(r, r^{\prime}, \beta\right)=\int \mathrm{d} x_{1} \ldots \mathrm{d} x_{N-1} \\
& \quad \times \rho^{\mathrm{bos}}\left(\left\{x_{1}, \ldots, x_{N-1}, r\right\},\left\{x_{1}, \ldots, x_{N-1}, r^{\prime}\right\}, \beta\right),
\end{aligned}
$$

is also a sum of permutation-dependent terms, and the Landsberg recursion relation can be generalized to a nondiagonal single-particle density matrix $\rho\left(r, r^{\prime}, k \beta\right)$ rather than to the diagonal one:

$$
\begin{aligned}
\rho_{N}\left(r, r^{\prime}, \beta\right) & =\frac{1}{N} \sum_{k=1}^{N} Z_{N-k} \rho\left(r, r^{\prime}, k \beta\right), \\
\frac{V}{Z_{N}} \rho_{N}\left(r, r^{\prime}, \beta\right) & =\sum_{k=1}^{N} \pi_{k} R_{k}^{\text {cut }}\left(r, r^{\prime}\right),
\end{aligned}
$$

where $V$ is the volume of the system. In eqn (8), the cycle probabilities $\pi_{k}$ are modified by the cut-off function $R_{k}^{\text {cut }}\left(r, r^{\prime}\right)$, which is proportional to the ratio of the offdiagonal and the diagonal density matrices. In the threedimensional homogeneous Bose gas in a periodic cubic box of size $L^{3}$, we have $R_{k}^{\text {cut }}\left(r, r^{\prime}\right)=L^{3} \rho\left(r, r^{\prime}, k \beta\right) / z_{k}=$ $\rho\left(0, r-r^{\prime}, k \beta\right) / \rho(0,0, k \beta)$.

As is well known, the condensate fraction is proportional to the off-diagonal single-particle density matrix in the limit $\left|r-r^{\prime}\right| \rightarrow \infty$, a case that corresponds to $\left|r-r^{\prime}\right| \propto L$ in a cubic box of length $L$ for $L \rightarrow \infty$. The cut-off function then vanishes for small $k$, and terms with $k \gg \frac{L^{2}}{2 \beta}$ dominate the sum in eqn (8) [8].

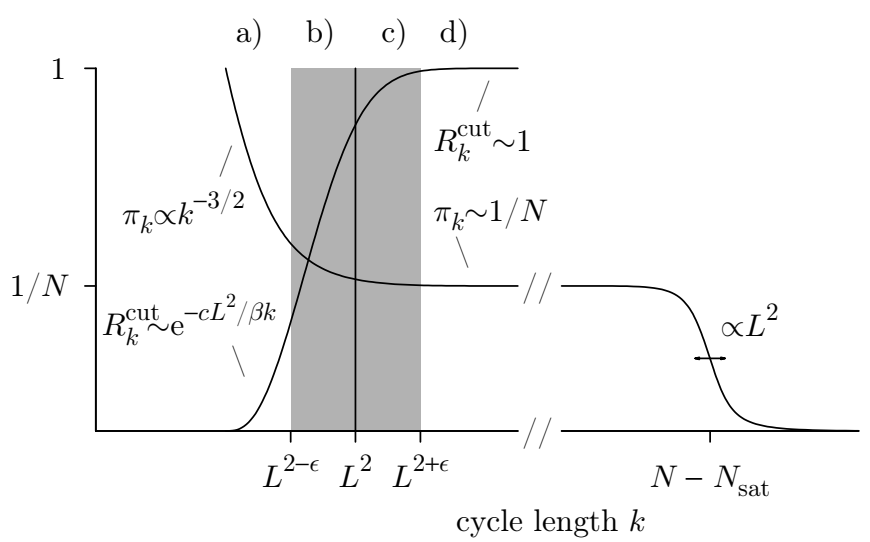

FIG. 1: Cycle probabilities $\pi_{k}$ and cut-off function $R_{k}^{\text {cut }}$ for a periodic box of size $L^{3}$ below $T_{c}$.

\section{OFF-DIAGONAL LONG-RANGE ORDER AND CYCLE PROBABILITIES}

In this section, we discuss a relation between the offdiagonal long-range order parameter and the sum of cycle probabilities for the homogeneous Bose gas, with unit mass and particle density $\left(m=\hbar=1, N=L^{3}, T_{c}=\right.$ $\left.2 \pi / \zeta(3 / 2)^{2 / 3}\right)$. To analyze eqn (8), we notice that $z_{k} \sim 1$ for $k \gg \frac{L^{2}}{2 \beta}$ and $z_{k} \sim L^{3} /(2 \pi \beta k)^{3 / 2}$ for $k \ll \frac{L^{2}}{2 \beta}$. It follows from $Z_{N-k}<Z_{N}$ that

$$
\pi_{k} \lesssim \begin{cases}1 /(2 \pi \beta k)^{3 / 2} & \text { for } k \ll \frac{L^{2}}{2 \beta} \\ 1 / N & \text { for } k \gg \frac{L^{2}}{2 \beta} .\end{cases}
$$

We now study the cut-off function $R_{k}^{\text {cut }}\left(r, r^{\prime}\right)$ for $\mid r-$ $r^{\prime} \mid \propto L$. For concreteness, we suppose that the vector $r-r^{\prime}$ has the same components in all three space directions: $r-r^{\prime}=L(\delta, \delta, \delta)$ with $0<\delta<\frac{1}{2}$ and denote the corresponding cut-off function and the off-diagonal density matrix by $R_{k}^{\text {cut }}(\delta)$ and by $\rho_{N}(\delta)$, respectively. We have

$$
R_{k}^{\text {cut }}(\delta)=\left[\frac{\sum_{w \in \mathbb{Z}} e^{-(\delta+w)^{2} L^{2} /(2 k \beta)}}{\sum_{w \in \mathbb{Z}} e^{-w^{2} L^{2} /(2 k \beta)}}\right]^{3} .
$$

For $k \gg \frac{L^{2}}{2 \beta}$, both the numerator and the denominator are $\sim \sqrt{2 \pi \beta k} / L$, so that $R_{k}^{\text {cut }}(\delta) \sim 1$. For $k \ll \frac{L^{2}}{2 \beta}$, the numerator is $\sim \exp \left\{-3 \delta^{2} L^{2} /(2 \beta k)\right\}$, and the denominator is $\sim 1$. This shows that the monotonic function $R_{k}^{\text {cut }}(\delta)$ is exponentially small for $k \ll \frac{L^{2}}{2 \beta}$ and equal to unity for $k \gg \frac{L^{2}}{2 \beta}$ (see Fig. 1). The estimates for $R_{k}^{\text {cut }}$ and for $\pi_{k}$ imply

$$
\begin{aligned}
& \lim _{L \rightarrow \infty} \sum_{k=1}^{L^{2}} \pi_{k} R_{k}^{\mathrm{cut}}(\delta)=0 \\
& \lim _{L \rightarrow \infty} \sum_{k=L^{2}}^{N} \pi_{k}\left[1-R_{k}^{\mathrm{cut}}(\delta)\right]=0 .
\end{aligned}
$$


(see the appendix for a short, but rigorous derivation). It follows that

$$
\lim _{L \rightarrow \infty}\left[\frac{L^{3} \rho_{N}(\delta)}{Z_{N}}-\sum_{k=\text { const } L^{2}}^{N} \pi_{k}\right]=0 .
$$

This equation relates the probabilities of infinite cycles to off-diagonal long-range order and (because $\lim _{\left|r-r^{\prime}\right| \rightarrow \infty} \frac{V}{Z_{N}} \rho_{N}\left(r, r^{\prime}, \beta\right)$ gives the condensate fraction) also to the condensate fraction. The relation between cycle lengths and off-diagonal long-range order is natural because, in order for a cycle to contribute to the off-diagonal density matrix for $\left|r-r^{\prime}\right| \propto L$, its de Broglie wavelength must be at least comparable to the distance $L$, which means that the particle must belong to a cycle of length $k$ with $L \lesssim \sqrt{2 \pi k \beta}$ (see also [2,9] for related derivations).

\section{CONDENSATE FRACTION AS A DERIVATIVE OF THE CYCLE PROBABILITIES}

In Section III, we studied the sum of the cycle probabilities. We now discuss the fact that the discrete derivative of the cycle probabilities exactly gives the condensation probabilities. This relation allows to compute the $\pi_{k}$, and it involves neither a cut-off function nor the thermodynamic limit and holds for arbitrary systems of ideal bosons. The relation was mentioned very briefly in the context of Monte Carlo calculations [4], but it was not analyzed in any detail.

Let us define $S_{N}(k, \sigma)$ as the canonic partition function with $N$ bosons of which exactly $k$ are in state $\sigma$ (see [6]) and, similarly, the restricted partition function with at least $k$ bosons in the state $\sigma$, as

$$
Y_{N}(k, \sigma)=\sum_{k^{\prime}=k}^{N} S_{N}\left(k^{\prime}, \sigma\right)=\mathrm{e}^{-\beta k \epsilon_{\sigma}} Z_{N-k} .
$$

$\left(S_{N}(k, \sigma) / Z_{N}\right.$ is the probability of having exactly $k$ bosons in state $\sigma$.) Summing eqn (12) over all states $\sigma$, and dividing by $N Z_{N}$, we recover the cycle probabilities,

$$
\frac{1}{N Z_{N}} \sum_{\sigma} Y_{N}(k, \sigma)=\frac{\sum_{\sigma} \mathrm{e}^{-\beta k \epsilon_{\sigma}}}{N Z_{N}} Z_{N-k}=\pi_{k}
$$

whose negative discrete derivative,

$$
\pi_{k}-\pi_{k+1}=\sum_{\sigma} S_{N}(k, \sigma) /\left(N Z_{N}\right),
$$

thus yields the sum over all $\sigma$ of the probabilities of condensing $k$ bosons into state $\sigma$. We note that the l.h.s. of eqn (13) contains only quantities related to the pathintegral picture, whereas the r.h.s. involves only energy levels. Furthermore, we note that the probability of condensing $k$ particles into excited levels is zero for large $k$, so that eqn (13) effectively relates the groundstate

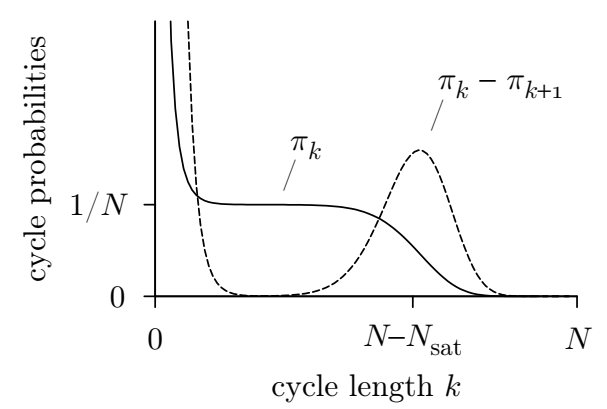

FIG. 2: Cycle probabilities $\pi_{k}$ and their negative discrete derivative (not to scale) for $N=125$ ideal bosons in a periodic box of size $L^{3}=5^{3}$ at temperature $T / T_{c}=0.5\left(N_{\text {sat }}=48.7\right)$.

condensation probabilities to the derivative of the cycle probabilities.

To illustrate the relation between cycles and condensation probabilities, we consider the example of Fig. 2 of $N$ particles in a periodic box, with the cycle probabilities explicitly computed from the Landsberg recursion relation. For $k>N / 2$, the derivative of the cycle probabilities equals the sum of the probabilities of condensing $k$ bosons into state $\sigma$. More generally, for $k \gg \frac{L^{2}}{2 \beta}$, the condensation probabilities into the excited states vanish and $\pi_{k}-\pi_{k+1} \sim S_{N}(k, 0) /\left(N Z_{N}\right)$ directly yields the probability distribution of having $k$ particles in the groundstate, a distribution with mean value $k=\left\langle N_{0}\right\rangle$ and standard deviation $\propto L^{2}$. On the other hand, for small $k$, as already discussed, the discrete derivative behaves as $k^{-5 / 2}$. It describes the probabilities of condensing $k$ bosons into excited states. Finally, for $\frac{L^{2}}{2 \beta} \ll k$ and $k \leq\left\langle N_{0}\right\rangle-L^{2} /(2 \pi \beta)^{3 / 2}$, the probability of having $k$ particles in the condensate vanishes, so that the $\pi_{k}$ are constant.

\section{DIRECT CALCULATION OF CYCLE PROBABILITIES}

We have seen in Section IV that the cycle probabilities $\pi_{k}$ follow from the known fluctuations of the condensate in the canonic ensemble. It is very instructive to compute them directly via the infinite-density limit for the partition function of an arbitrary system. For $k \gg \frac{L^{2}}{2 \beta}$, we have $z_{k} \sim 1$, so that $\pi_{k} \sim Z_{N-k} / Z_{N}$. For illustration, the Fig. 3 shows the $Z_{k}$ in a fixed physical system (for the homogeneous Bose gas, in a fixed box of size $L^{3}$ ), as it can be computed numerically from the Landsberg recursion relation. The limiting value $Z_{\infty}$ (at infinite density) and the value $k=N_{\text {sat }}$ of largest variation of $Z_{k}$ can be computed exactly.

Indeed, as the groundstate energy is zero, $Z_{N}$ differs from $Z_{N-1}$ only by configurations without any particle in the groundstate: $Z_{N}-Z_{N-1}=S_{N}(0,0)$ (see above 


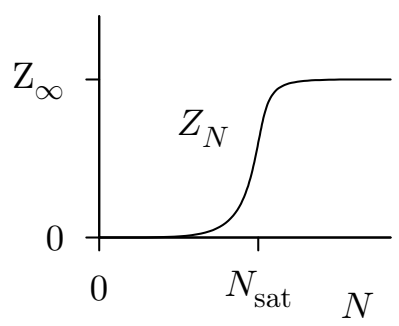

FIG. 3: Canonic partition function $Z_{N}$ as a function of $N$ in a finite system.

eqn (12)). Therefore, $Z_{N}=\sum_{k=1}^{N} S_{k}(0,0)$ with

$$
S_{k}(0,0)=\left[\prod_{\sigma>0} \sum_{n_{\sigma}=0}^{\infty}\right] \exp \left(-\beta \sum_{\sigma>0} n_{\sigma} \epsilon_{\sigma}\right) \delta_{\sum_{\sigma>0} n_{\sigma}, k} .
$$

Summing this equation over all integers $k$ yields, for arbitrary states $\left\{\epsilon_{\sigma}\right\}$,

$$
Z_{\infty}=\prod_{\sigma>0} \frac{1}{1-\exp \left(-\beta \epsilon_{\sigma}\right)}=\exp \left(\sum_{k=1}^{\infty} \frac{z_{k}-1}{k}\right) .
$$

This exact formula for a finite canonical Bose system can be obtained without the usual saddle-point integration. It agrees with the grand-canonical partition function for the excited states at zero chemical potential because at high density the condensate serves as a reservoir for the excited bosons.

To determine the value of $N$ for which the partition function $Z_{k}$ passes from $Z_{k} \sim 0$ to $Z_{k} \sim Z_{\infty}$ (see Fig. 3), we again take a discrete derivative and consider the probability distribution of the number of excited particles

$$
\pi_{\infty}\left(N_{\mathrm{exc}}=k\right)=\left(Z_{k}-Z_{k-1}\right) / Z_{\infty},
$$

whose mean value, the saturation number, is

$$
\begin{aligned}
& N_{\mathrm{sat}}=\left\langle N_{\mathrm{exc}}\right\rangle=\sum_{k=1}^{\infty} k \pi_{\infty}\left(N_{\mathrm{exc}}=k\right)= \\
& \sum_{\sigma>0} \frac{\exp \left(-\beta \epsilon_{\sigma}\right)}{1-\exp \left(-\beta \epsilon_{\sigma}\right)}=\sum_{k=1}^{\infty}\left(z_{k}-1\right) .
\end{aligned}
$$

The variance of the number of excited particles is again given exactly by the corresponding grand-canonical expressions

$$
\begin{aligned}
\left\langle N_{\text {exc }}^{2}\right\rangle-\left\langle N_{\text {exc }}\right\rangle^{2}= & \sum_{\sigma>0} \frac{1}{\exp \left(\beta \epsilon_{\sigma}\right)-1}+\frac{1}{\left[\exp \left(\beta \epsilon_{\sigma}\right)-1\right]^{2}} \\
& =N_{\text {sat }}+\sum_{k=2}^{\infty}(k-1)\left(z_{k}-1\right) .
\end{aligned}
$$

For completeness, we give the expressions corresponding to eqn (14)-eqn (16) in the homogeneous Bose gas, in the limit $L \rightarrow \infty: Z_{\infty} \sim \exp \left[L^{3} /(2 \pi \beta)^{-5 / 2} \zeta(5 / 2)\right]$ and, furthermore, $N_{\text {sat }} / L^{3} \sim \zeta(3 / 2) /(2 \pi \beta)^{3 / 2}=\eta_{c}$, with $\eta_{c}$ the critical density. In addition, the variance of $N_{\text {exc }}$ behaves as $L^{4} /(2 \pi \beta)^{3}[10]$, so that the jump of $Z_{k} / Z_{N}$ from 0 to 1 , for $T<T_{c}$, takes place in a window of width $L^{2}$, as already noted in Section IV.

Putting together the analysis of $Z_{k}$ for a fixed size of the system and a varying particle number, and our knowledge of $z_{k}$, we have a complete description of the cycle probabilities $\pi_{k}$. Once we know exactly the behavior of the $\pi_{k}$ in the thermodynamic limit, we observe that the sum of the $\pi_{k}$ for $k \ll \frac{L^{2}}{2 \beta}$ gives exactly the critical density $\eta_{c}$, and the sum of the $\pi_{k}$ for $k \gg \frac{L^{2}}{2 \beta}$ gives the condensate fraction. This conclusion is the same as in Section III, but it stems from a microscopic analysis of the cycle probabilities.

\section{MAXIMUM CYCLE LENGTHS}

At finite temperature, the cycle probabilities $\pi_{k}$ are easily computed from the Landsberg recursion relation, and at $T=0$, they are given by $\pi_{k}=1 / N$. In Monte Carlo calculations the entire permutation of $N$ elements can be sampled, starting from the cycle containing the element $N$, which has length $k$ with probability $\pi_{k}$. This leaves one with a system of $N-k$ particles, for which the probabilities of the cycle containing element $(N-k)$ can again be computed, etc. (see [7]). (At zero temperature, the distribution remains $\pi_{k}=1 /(N-k)$, at finite temperature, it is given by eqn (6).)

In this section, we use the cycle probabilities $\pi_{k}$ to obtain analytical results for $p_{k}^{\max }$, the probability that the longest cycle in a permutation has length $k$. This probability distribution is closely related to the distribution $\pi_{k}$ both at finite temperature and at $T=0$, where the ideal Bose gas is equivalent to the problem of random permutations, which has been much studied in the mathematics literature. We recover some classic results which we generalize to finite temperatures.

We first note that the cycle probabilities are related to the mean number of cycles of length $k, \tilde{\pi}_{k}=N \pi_{k} / k$. For $k>N / 2, \tilde{\pi}_{k}$ coincides with the probability $p_{k}$ that the permutation $P$ has at least one cycle of length $k$. This is simply because a permutation of $N$ elements can have no more than one such cycle, in other words, because

$$
\tilde{\pi}_{k}=\sum_{m=1}^{\infty} m \times\left\{\begin{array}{c}
\text { prob. to have } \\
m \text { cycles of length } k
\end{array}\right\}
$$

and

$$
p_{k}=\sum_{m=1}^{\infty}\left\{\begin{array}{c}
\text { prob. to have } \\
m \text { cycles of length } k
\end{array}\right\},
$$

and only terms with $m=1$ contribute to the above expressions if $k>N / 2$. 
Furthermore, any cycle of length $k>N / 2$ must be the longest cycle, so that we arrive for all temperatures at:

$$
p_{k}^{\max }=p_{k}=\tilde{\pi}_{k}=N \pi_{k} / k \quad \text { for } k>N / 2, \forall T .
$$

We next consider the probability distributions $p_{k}$ and $p_{k}^{\max }$ for $k \leq N / 2$ at $T=0$, for $N \rightarrow \infty$. We take the continuum limit $p_{k} \rightarrow p(x)$ by setting $p_{k}=\frac{1}{N} p\left(x=\frac{k}{N}\right)$ (and similarly for $p^{\max }(x)$ and $\tilde{\pi}(x)$ ).

It follows from the recursive procedure, and from the fact that the $\pi_{k}$ are of order $1 / N$, that the probability to have more than one cycle of the same length is $O\left(1 / N^{2}\right)$. In the limit $N \rightarrow \infty$, we see that $p(x)=\tilde{\pi}(x)=1 / x$.

For $x<1 / 2$, the probability $p^{\max }(x)$ is the product of the probability to sample $x$, and the probability that $x$ remains the longest cycle in the remaining partition of length $1-x$, in other words:

$$
p^{\max }(x)=p(x)\left(1-\left\{\begin{array}{c}
\text { prob. that longest cycle in } \\
{[0,1-x] \text { is }>x}
\end{array}\right\}\right) .
$$

At $T=0$, and in the limit $N \rightarrow \infty$, the probability that in a permutation of length $N(1-x)$ the longest cycle's length exceeds $N x$ equals the probability that in a permutation of $N$ elements the longest cycle exceeds $x /(1-x)$, and we arrive at

$$
p^{\max }(x)=p(x)\left[1-\int_{x /(1-x)}^{1} \mathrm{~d} x^{\prime} p^{\max }\left(x^{\prime}\right)\right] .
$$

For all $x \in] 0,1[, x<x /(1-x)$, so eqn $(20)$ can be used to compute $p^{\max }(x)$ from $x=1$ downwards to arbitrary precision (see Fig. 4). Alternatively, we can transform eqn (20) into a differential equation

$$
\frac{d}{d x} p^{\max }(x)=-\frac{1}{x} p^{\max }(x)+\frac{1}{x(1-x)^{2}} p^{\max }\left(\frac{x}{1-x}\right) .
$$

Explicit formulas for $p^{\max }(x)$ are easily obtained, starting by inputting, from eqn (19), $p^{\max }(x)$ for $\frac{1}{2}<x<1$ to obtain $p^{\max }$ in the window $x \in\left[\frac{1}{3}, \frac{1}{2}\right]$, etc,

$$
p^{\max }(x)=\left\{\begin{array}{l}
\frac{1}{x} \text { for } \frac{1}{2}<x \\
\frac{1}{x}\left[1-\log \left(\frac{1-x}{x}\right)\right] \text { for } \frac{1}{3}<x<\frac{1}{2},
\end{array}\right.
$$

but they become cumbersome (see also [11]).

At finite temperature, the cycle of the element $N$ is sampled from a distribution $\pi_{k}$ as shown in Fig. 2 rather than from the flat distribution. However, the longest cycle is again of length $\propto N$, and it is sampled from an essentially constant distribution in the interval $x \in$ $\left[0,\left\langle N_{0}\right\rangle / N\right]$. In the thermodynamic limit, for $T<T_{c}$, the distribution $p^{\max }(x)$ of the properly rescaled variable $x=k^{\max } /\left\langle N_{0}\right\rangle$ trivially agrees with the one obtained at $T=0$. In particular, the mean length of the longest cycle is given by $0.624\left\langle N_{0}\right\rangle$ at all temperatures $T<T_{c}[5]$. At sufficiently low temperature, the rounding at $x \simeq 1$ is exactly described by eqn (19) (see Fig. 4).

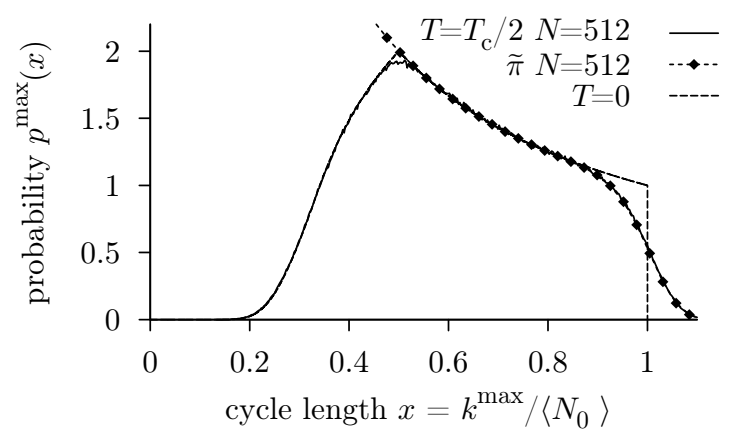

FIG. 4: Probability $p^{\max }(x)$ from a numerical integration of eqn (20) at $T=0$, compared to the distribution $p_{k}^{\max }$ obtained from a Monte Carlo sampling at $T=T_{c} / 2$, and the exact expression of eqn (19) for large $x$, at $T=T_{c} / 2$.

\section{INTERACTING BOSE GAS}

For the interacting Bose gas, the relation between offdiagonal long-range order and the infinite cycles has been studied for generalized mean-field models $([2,12])$, but it has proven difficult to establish rigorous results for models with realistic interactions. In the general case, the definition of the superfluid density in terms of the winding numbers [13] proves that for the homogeneous Bose gas in three dimensions, the presence of infinite cycles is equivalent to a non-zero superfluid density. It is generally admitted that in homogeneous systems, BoseEinstein condensation implies superfluidity, so that the presence of a condensate would imply the existence of infinite cycles in those systems.

On the other hand, the direct link between the cycle probabilities and the condensate fraction, as described in Section IV, cannot hold for interacting systems. This is easily seen at $T=0$ because the groundstate of any system of identical but distinguishable particles has bosonic symmetry. Therefore, the distinguishable-particle density matrix

$$
\rho\left(\left\{x_{1}, \ldots, x_{N}\right\},\left\{x_{P_{1}}, \ldots, x_{P_{N}}\right\}, \beta=\infty\right)
$$

is independent of the permutation $P$. It follows that at $T=0$, and for finite $N$, all permutations have the same weight and the cycle probabilities again satisfy $\pi_{k}=\frac{1}{N}$. At the same time, the condensate fraction of interacting systems at $T=0$ is less than one[14], and the distribution of the $\pi_{k}$ cannot be related to the distribution of $N_{0}$ in the same manner as in the ideal Bose gas.

\section{CONCLUSION}

In conclusion, we have discussed the relation between the off-diagonal long-range order, the condensate fraction, and the cycle probabilities in the ideal Bose gas. Only long cycles $\left(k>\operatorname{const} L^{2}\right.$ in the homogeneous case) 
contribute to the off-diagonal long-range order parameter, and Bose Einstein condensation is equivalent to the presence of infinite cycles. We have also discussed the probability distribution for long cycles, and the general, non-intuitive, link between the integer derivative of the cycle probabilities and the number of condensed bosons. This integer derivative provides us with a purely topological characterization of the condensate fraction in the ideal Bose gas. Our knowledge of the cycle probabilities allows us to study the distribution of the longest cycle. We have remarked that the $0<T<T_{c}$ case can be understand from the $T=0$ case. The mean length of the longest cycle is proportional to the number of condensed bosons. For the interacting Bose gas, at the present time, only the winding-number formula[13] has been rigorously shown to relate the topology of Feynman paths to another characteristic of interacting Bose-condensed systems, namely the superfluid density. The link between Bose-Einstein condensation and superfluidity will need to be explored further in order to better understand the cycles of the interacting Bose gas.

\section{Acknowledgments}

We thank A. Comtet and S. N. Majumdar for an inspiring discussion about maximum cycle length (see also
[15]) and for making us aware of the papers $[5,11]$.

\section{APPENDIX A: DERIVATION OF EQN (10)}

In this appendix, we prove eqn (10). We consider the sums separately in the regions a) - d) indicated in Fig. 1 and show that they all vanish individually.

In region a), $\pi_{k} R_{k}^{\text {cut }}(\delta) \lesssim \pi_{k} \exp \left[-3 \delta^{2} L^{\epsilon} / \beta\right]$, and the normalization of the $\pi_{k}$ shows that the partial sum vanishes in the limit $L \rightarrow \infty$. In b), $\pi_{k} R_{k}^{\text {cut }} \leq$ $\pi_{L^{2-\epsilon}} R_{L^{2}}^{\text {cut }} \lesssim$ const $/ L^{3(2-\epsilon) / 2}$ and the partial sum is smaller than const $L^{3 \epsilon / 2-1}$. In c), as $1-R_{k}^{\text {cut }}(\delta)$ decreases with $k, \pi_{k}\left[1-R_{k}^{\text {cut }}(\delta)\right] \leq \pi_{L^{2}}\left[1-R_{L^{2}}^{\text {cut }}(\delta)\right]$ and the partial sum is dominated by const $L^{\epsilon-1}$. Finally, in d), $\pi_{k}\left[1-R_{k}^{\text {cut }}(\delta)\right] \leq \pi_{k}\left[1-R_{L^{2+\epsilon}}^{\text {cut }}(\delta)\right]$. The normalization condition of the $\pi_{k}$ and the relation $L^{2+\epsilon} \gg L^{2}$ imply that this partial sum vanishes for $L \rightarrow \infty$. With a suitable choice of $\epsilon$ (for example $\epsilon=\frac{1}{2}$ ), these sums all vanish for $L \rightarrow \infty$. In fact, any function $\epsilon(L) \gg 1 / \log L$ that satisfies $\epsilon(L)<2 / 3$ for $L>L_{0}$ can be used.
[1] R. P. Feynman, Statistical Mechanics (Addison-Wesley, New York, 1972).

[2] D. Ueltschi, J. Math. Phys. 47, 123303 (2006).

[3] G. Benfatto, M. Cassandro, I. Merola, and E. Presutti, J. Math. Phys. 46, 033303 (2005).

[4] M. Holzmann and W. Krauth, Phys. Rev. Lett. 83, 2687 (1999).

[5] L. A. Shepp and S. P. Lloyd, Trans. Amer. Math. Soc. 121, 340 (1966).

[6] See appendix E of P. T. Landsberg, Thermodynamics, with quantum statistical illustrations (Interscience, New York, 1961).

[7] W. Krauth, Statistical Mechanics: Algorithms and Computations (Oxford University Press, Oxford, 2006).

[8] We use the notations $A \sim B \Leftrightarrow \lim _{L \rightarrow \infty} \frac{A}{B}=1 ; A \ll$
$B \Leftrightarrow \lim _{L \rightarrow \infty} \frac{A}{B}=0 ; A \gg B \Leftrightarrow \lim _{L \rightarrow \infty} \frac{A}{B}=+\infty ;$ $A \propto B \Leftrightarrow \lim _{L \rightarrow \infty} \frac{A}{B}=$ const $>0$. Furthermore, $A \lesssim$ $B \Leftrightarrow \lim _{L \rightarrow \infty} \frac{A}{B} \leq 1$.

[9] A. Suto, J. Phys. A: Math Gen 35, 6995 (2002).

[10] R. M. Ziff, G. E. Uhlenbeck, and M. Kac, Phys. Rep. 32 169 (1977).

[11] V. Goncharov, C. R. Acad. Sci. URSS (N.S) 35, 267 (1942).

[12] T. C. Dorlas, P. A. Martin, J. V. Pule, J. Stat. Phys.121, 433 (2005).

[13] E. L. Pollock and D. M. Ceperley, Phys. Rev. B 36, 8343 (1987).

[14] O.Penrose, Phys. Lett. 11 (3): 224-226 (1964).

[15] A. Comtet and S. N. Majumdar, unpublished. 